\title{
Low-risk Thyroid Cancer, Surgical Training, and Radioactive Iodine
}

\author{
Ashok R. Shaha, MD \\ Department of Surgery, Memorial Sloan-Kettering Cancer Center, New York, NY
}

Schuessler ${ }^{1}$ and her colleagues from the University of Michigan have undertaken an interesting and complex study of surgeon training and use of radioactive iodine for low risk thyroid cancer patients. This study is based on several hypotheses, a questionnaire to the surgeons and correlating their training to radioactive iodine use. Clearly, management of thyroid cancer starting from extent of thyroidectomy, radioactive iodine use and extent of suppressive therapy continue to be a controversial subject and depends on experience and judgment of the treating physician. Even though there are no firm standards of practice, the general tendency in the United States appears to be total thyroidectomy followed by radioactive iodine ablation. Some of these decisions are made definitely by surgeons who have performed the surgical procedure; however, there is an enormous impact of endocrinologists, and the patient themselves having read extensively on Google. Clearly, trying to correlate these issues of treatment, referring specifically to the surgeon training, parent specialty, and attending the professional meeting may be difficult. In any case, the authors have made a genuine effort to correlate these issues and concluded that training with a thyroid surgeon and attending one or more professional society meeting a year was associated with lower rate of hospital based RAI use. Clearly, these conclusions may be difficult to digest because decision making regarding RAI is generally not one individual decision. The manuscript mentions Stage I thyroid cancer patients while a majority of the manuscript discusses the low risk thyroid cancer patients. Clearly, Stage I thyroid cancer patients,

(C) Society of Surgical Oncology 2012

First Received: 9 November 2012; Published Online: 20 December 2012

A. R. Shaha, MD

e-mail: shahaa@mskcc.org especially below the age of 45 with lymph node metastasis, definitely will require radioactive iodine and is quite rational approach, while in the truly low risk thyroid cancer patient, where the tumor appears to be intrathyroidal, the role of radioactive iodine is always questioned and similar opinion is reflected in the ATA guidelines. The basic conclusion that can be made out of this manuscript is the surgeon training, and the experience of the surgeon is more important in treatment policies.

Having read this manuscript several times, certain important points need to be highlighted. The collection of the data and its relation to NCDB is quite complex. It should be mentioned that the average surgeon age was 51 years and the surgeon's average years in practice was 19. This reflects the seniority of the surgeons and their training several years before the ATA guidelines and our recent multidisciplinary management of thyroid cancer.

The practice distribution of thyroidectomy is also quite interesting that the general surgeons comprised of $39 \%$ while the otolaryngologists comprised of $44 \%$ a reflection of current shifting practice pattern in the United States. There are very few individuals who are members of the American Association of Endocrine Surgeons with high level training background of the endocrine surgery. The authors have mentioned about $63 \%$ individuals attending one or more professional society meetings per year. This information becomes extremely vague and difficult to interpret as what type of professional society meeting is considered. It remains unclear whether this is a national society or local organization. It is also uncertain as to how much of the thyroid discussion is undertaken in these society meetings.

It is interesting and somewhat concerning that only over half of the respondents reported reading 2006 ATA guidelines. Over the last 6 years, ATA guidelines have become important decision makers and both the endocrinologist and surgeons involved in thyroid cancer 
management refer to ATA guidelines frequently. Clearly, the decision making in the use of radioactive iodine depends on the understanding of the prognostic factors and risk group analysis in thyroid cancer. ${ }^{2,3}$ There is hardly any data that the routine use of radioactive iodine in low risk thyroid cancer patients has shown any major impact either on local recurrence, nodal metastasis or distant metastasis. However, ample data are available regarding the side effects of routine use of radioactive iodine such as xerostomia, and slightly higher incidence of development of second primary tumors. ${ }^{4}$ Even though recombinant thyroid-stimulating hormone (rTSH) is available in the last few years the majority of the respondents in this analysis probably did not use rTSH and thyroid hormone withdrawal in patients has direct relation to the quality of life of these patients in the postoperative period. In recent years, the majority of patients with thyroid cancer are being followed by endocrinologists and even though the surgeons do make initial decisions about surgery, radioactive iodine, etc, the endocrinologists do play a major role in treatment decisions in these patients and long-term follow-up.

The authors have made a point in their discussion that training with thyroid surgeon provides increased focused experience that instills greater confidence in surgical skills and a more sophisticated view of thyroid disease management. Clearly, the surgical skill and understanding of the thyroid cancer management is very important; however, the indications for radioactive iodine are based on the extent of the disease, risk group rather than the surgical skill itself. Clearly, the surgical skills will permit doing a good total thyroidectomy, reducing the complications rate related to nerve injury and hypoparathyroidism; however, the decision to administer radioactive iodine is mainly based on the extent of the disease rather than the surgical skill itself. In the authors' final remarks in their discussion, they note, "These results suggest that to initiate change in hospital practice patterns a potential for improved adherence to clinical guidelines; we need to focus on physician training." Obviously, this is an important remark in the management of any medical or surgical problem. Clearly, the surgeon training is extremely important be that during residency, or in the clinical practice. There is a strong insistence in recertification, hours of CME attendance. Development of multidisciplinary programs is important in making critical decisions in the management of thyroid cancer. The treating physician needs to understand the biology of the disease and make appropriate decisions about radioactive iodine only when it is necessary rather than making a knee-jerk reflex of routine use of radioactive iodine. It is important to avoid overtreatment in the management of low risk thyroid cancer and avoid treatment related medical and surgical complications.

Clearly training with well trained thyroid surgeons and thyroid cancer experts is important. It is also important for well trained thyroid cancer physicians to train their medical and surgical trainees to understand the biology, prognostic factors and risk group analysis leading to development of multidisciplinary team. There is a major focus on shifting away from routine use of radioactive iodine in low risk thyroid cancer patients, as the survival in this group exceeds $98 \% .^{5}$ Unfortunately, patients often ask for scans and ablation to make sure their tumor has not spread to other parts of the body and often insist on overtreatment. Clearly, such overtreatment has no sound biological basis and may have unpleasant sequelae. It is our responsibility to convince patients and their families about the biology and risk group stratification of the thyroid cancer. Let the punishment fit the crime.

\section{REFERENCES}

1. Schluesser KM, Banerjee M, Yang D, Stewart AK, Doherty GM, Haymart MR. Surgeon training and use of radioactive iodine in stage I thyroid cancer patients. Ann Surg Oncol. doi:10.1245/ s10434-012-2745-0.

2. Shaha AR. Implications of prognostic factors and risk groups in the management of differentiated thyroid cancer. Laryngoscope. 2004;114:393-402.

3. Tuttle RM, Leboeuf R, Shaha AR. Medical management of thyroid cancer: a risk adapted approach. J Surg Oncol. 2008;97:712-6.

4. Iyer NG, Morris LG, Tuttle RM, Shaha AR, Ganly I. Rising incidence of second cancers in patients with low-risk (T1N0) thyroid cancer who receive radioactive iodine therapy. Cancer. 2011;117:4439-46.

5. Hay ID. Selective use of radioactive iodine in the postoperative management of patients with papillary and follicular thyroid carcinoma. J Surg Oncol. 2006;94:692-700. 